\title{
SAURON dynamical modeling of NGC 2974
}

\author{
Davor Krajnović ${ }^{1}$, Michele Cappellari ${ }^{1}$, Eric Emsellem ${ }^{2}$, \\ Richard McDermid ${ }^{1}$, P. Tim de Zeeuw ${ }^{1}$ \\ 1 Sterrewacht Leiden, P.O.Box 9513, 2300 RA Leiden, The Netherlands \\ 2 CRAL, 9 Av. Charles-Andre, 69230 Saint Genis-Laval, France
}

\begin{abstract}
We present a three-integral orbit superposition Schwarschild model of NGC 2974, observed with the integral-field unit SAURON. We explore the dynamical structure of the stars and gas.
\end{abstract}

\section{Introduction}

NGC 2974 is a nearby field elliptical galaxy classified as E4 with detected HI and $\mathrm{H} \alpha$ gas apparently distributed in a disk (Kim et al. 1988; Buson et al. 1993). An earlier study by Cinzano \& van der Marel (1994) suggested the galaxy harbours an embedded stellar disk not visible in the photometry. The galaxy was observed with the integral-field spectrograph SAURON (Bacon et al. 2001) mounted on the $4.2 \mathrm{~m}$ WHT on La Palma, as a part of the survey of a representative sample of nearby E, S0 and Sa galaxies (de Zeeuw et al. 2002). With this individual study we explore the dynamical structure of the object, give a quantitative analysis of the embedded disk structure and investigate the distribution and dynamics of the gas in NGC 2974. A similar analysis is being performed on the galaxies of the sample, providing constraints on galaxy formation scenarios.

\section{Stellar dynamical model}

The SAURON observations of emission-lines are consistent with the gas being distributed in a circular disk, except in the inner $3^{\prime \prime}$ where a bar-like perturbation was detected (Emsellem, Ferruit, \& Goudfrooij 2003). By assuming the gas is in a thin disk and fitting to the two-dimensional kinematics we are able to constrain the inclination of the disk to $i \sim 60^{\circ}$. Adopting that inclination we construct an axisymmetric model for the galaxy stellar body using a three-integral orbit superposition Schwarzschild modeling technique adapted for integral field data (e.g. Cretton et al. 1999, Cappellari et al. 2002, Verolme et al. 2002).

The best fitting model (Fig. 1) is able to reproduce all the features of the observed stellar kinematics. Analyzing the orbital distribution of the model we find two clearly distinct kinematic components. One component consists of high angular momentum orbits, producing a flattened density distribution, and the other component is rounder and rotates slowly. We associate the former component with a stellar disk and the latter with the spheroid. It is possible to perform a disk-bulge decomposition in phase space and estimate their mass fraction which turns out to be about 1:3. 


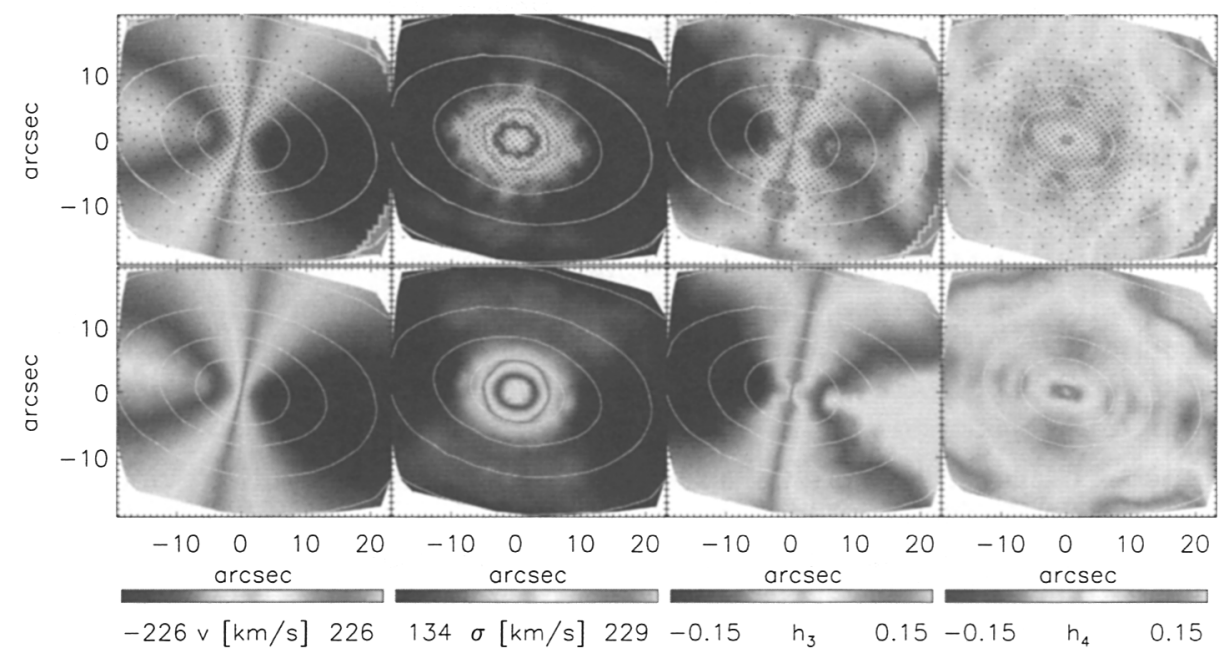

Figure 1. Symmetrized SAURON data of NGC 2974 and the best fitting dynamical model; from left to right: stellar mean velocity $(v)$, velocity dispersion $(\sigma)$, Gauss-Hermite moments $h_{3}$ and $h_{4}$. First row: SAURON data filtered by means of Fourier expansion with enforced bisymmetry as a visual aid for comparison of the data to the axisymmetric model. Original Voronoi 2D-binned data used to constrain the model can be found in Emsellem et al. (2003). Centroids of the bins are shown with dots. Second row: prediction from the best fitting regularized orbit superposition dynamical model at $i=60^{\circ}$ and $M / L=3.9$ (I-band).

We construct a dynamical model for the gas with the same potential and inclination used for the stellar model. Solving the Jeans equations in the limit of the asymmetric drift approximation we are able to reproduce the large scale gas kinematics providing an independent consistency test of the potential used for the stellar modeling.

\section{References}

Bacon, R. et al. 2001, MNRAS, 326, 23

Buson, L.M. et al. 1993, A\&A, 280, 409

Cappellari, M. et al. 2002, ApJ, 578, 787

Cinzano, P., \& van den Marel, R.P. 1994, MNRAS, 270, 325

Cretton, N., de Zeeuw, P.T, van der Marel, R.P, \& Rix, H. 1999, ApJS, 124, 383

de Zeeuw, P.T. et al. 2002, MNRAS, 329, 513

Emsellem, E., Ferruit, P., \& Goudfrooij, P. 2003, A\&A, in press

Emsellem, E. et al. 2003, MNRAS, submitted

Kim, D.W. et al. 1988, ApJ, 330, 684

Verolme, E.K. et al. 2002, MNRAS, 335, 517 